\title{
Self-assessment questions: Acute treatment and prevention of stroke
}

\author{
Philip MW Bath, Sandeep Ankolekar, Tim J England and Nikola Sprigg
}

1 In patients with acute/recent stroke:
(a) High blood pressure (BP) is present in $60 \%$ of patients
(b) Antihypertensive agents reduce cerebral blood flow
(c) Nitric oxide donors reduce vascular compliance
(d) All nitric oxide donors inhibit platelet function
(e) Amphetamine increases BP

2 In patients with acute stroke:

(a) Blood pressure should be reduced if systolic BP $>160$ $\mathrm{mmHg}$

(b) Beta blockers are the preferred drug class to reduce BP

(c) Thrombolysis should be administered only if systolic BP $<185 \mathrm{mmHg}$

(d) Treating high BP reduces haemorrhagic transformation of ischaemic stroke

(e) Urinary retention may increase BP

3 In patients with acute stroke, GTN therapy:

(a) Reduces cerebral blood flow

(b) Inhibits platelet function and so is unsafe for use in intracerebral haemorrhage

(c) Can lead to rebound changes in BP after withdrawal

(d) Reduces systolic BP by $23 \mathrm{mmHg}$ over 24 hours

(e) Cannot be given to patients with dysphagia
4 For secondary prevention of ischaemic stroke or transient ischaemic attack (TIA):

(a) The combination of aspirin and clopidogrel is recommended for secondary prevention in high-risk patients

(b) Aspirin and dipyridamole are recommended by the National Institute for Health and Clinical Excellence (NICE) for secondary prevention after TIA

(c) Clopidogrel monotherapy is recommended for secondary prevention after TIA

(d) Aspirin and clopidogrel does not increase bleeding events when given in combination after ischaemic stroke

(e) Aspirin and dipyridamole given in combination are more effective than aspirin alone for secondary prevention after ischaemic stroke

5 Regarding antiplatelet agents:

(a) Aspirin reversibly inhibits cyclooxygenase

(b) Aspirin is primarily excreted by the kidney

(c) Dipyridamole inhibits phosphodiesterase and increases cyclic adenosine monophosphate in platelets

(d) Dipyridamole stimulates uptake of adenosine by red blood cells, thereby inhibiting platelet activity

(e) Clopidogrel is a prodrug and requires hepatic metabolism to generate the active metabolite

Answers to these self-assessment questions can be found on page 592 . 